\title{
A Wireless Visual Attention Brain Signal Monitoring System
}

\author{
Simone Massai ${ }^{1}$, Sudhamayee Routhu ${ }^{1}$, Dustin Wright ${ }^{1}$, Kee S. Moon ${ }^{1, a}$, Yusuf. Ozturk ${ }^{1}$, and Sung Q Lee ${ }^{2}$ \\ ${ }^{1}$ San Diego State University, California, USA \\ ${ }^{2} E T R I$, Daejon, S. Korea
}

\begin{abstract}
With the recent advances in the field of electroencephalography (EEG), researchers have been able to detect and predict cognitive states and patterns based on electromagnetic signals emitted by the brain with greater precision than ever before. EEG has become a viable means of implementing brain-computer-interfaces (BCIs), which translate brain signals into machine commands. We have developed a system with reconfigurable hardware and open architecture component based software to enable multi-sensor physiological signal monitoring. The goal of this research is to study the feasibility of using the system that can facilitate monitoring of visual attention EEG brain signal.
\end{abstract}

\section{Introduction}

Brain signals like electroencephalography (EEG) are used to access brain functions. EEG is typically non-invasive method to record electrical activity of the brain along the scalp. EEG measures voltage fluctuations resulting from ionic current within the neurons of the brain. In clinical contexts, EEG refers to the recording of the brain's spontaneous electrical activity over a period, as recorded from multiple electrodes placed on the scalp [1]. With the increased miniaturization of EEG systems, its use has spread to lifestyle and entertainment purposes. With the recent advances in the field of EEG, researchers have been able to detect and predict cognitive states and patterns based on electromagnetic signals emitted by the brain with greater precision than ever before. EEG has become a viable means of implementing brain-computer interfaces (BCIs), which translate brain signals into machine commands.

It is known that attention is the behavioral and cognitive process of selectively concentrating on a discrete aspect of information, while ignoring other perceivable information. Attention has also been referred to as the allocation of limited processing resources [2]. Attention remains a major area of investigation within education, psychology, neuroscience, cognitive neuroscience, and neuropsychology [3].

There have been different approaches to detecting brain attention (or concentration) through machine learning and EEG feature extraction methods [4-9]. The goal of the study is to develop an EEG-based visual attention detection system and implement active sensor hardware which can sense the small signals emitted by the brain while minimizing the useful signal to noise ratio (SNR). The result is a system, which can detect visual

\footnotetext{
a Corresponding author: kmoon@mail.sdsu.edu
}

attention levels in the users by comparing the relative energy levels of the alpha and beta bands while using this information to control mind-controlled devices.

\section{Multi sensor reconfigurable physiological signal monitoring system}

Physiological signal recording, transmission, collection and analysis play an important role not only in health care applications but can also find use in other applications such as physiological signal based games, interactive system designs that include physiological signals as primary control signals etc. The core of the signal monitoring depends on the type of signal being monitored and the location from which signal is observed. For example during collection of electromyography (EMG) signals, sensors will be placed on the muscle being monitored. Similarly, for EEG signals sensors are placed on the forehead while motion sensors are placed on the part of the body whose motion needs to be recorded.

Most of the physiological signal monitoring systems involve selection of the sensors, a front end analog to digital converter circuitry to digitize the signals and appropriate data communication circuitry to transfer monitored data to a display to a host processor for further analysis. Although each signal differs in amplitude and frequency, the core of the signal acquisition involves detection, digitization and communication of the signals to the host. By changing sensors and tuning the front electronics according to the signals being monitored, number of new physiological signals can be monitored. By exploiting the similarity involved in signal acquisition path, we have developed a new signals monitoring system. As shown in Figure 1, electrodes convert the

This is an Open Access article distributed under the terms of the Creative Commons Attribution License 4.0, which permits unrestricted use, distribution, and reproduction in any medium, provided the original work is properly cited. 
actual physiological signals into electrical potentials. These are then passed to a multiplexer for multiplexing between different sensors. Signals are then amplified and filtered. Filtered signals are digitized using analog to digital converter and are transmitted using a transmitter/receiver modules. Transmitted signals are captured by the other end like a PC or host where the digitized signals are further processed for artifact removal and advanced signal processing depending on the system. By varying the electrodes/sensors, tuning the amplifier gains, filter parameters variety of physiological signals can be monitored.

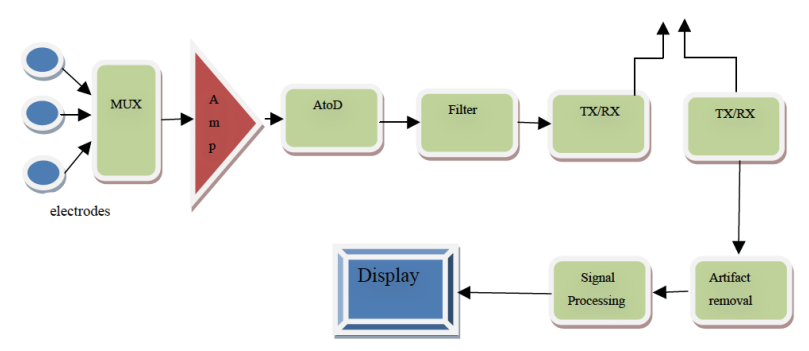

Figure 1. Reconfigurable physiological signal monitoring system.

We have employed modular hardware architecture with well-defined hardware components. The developed system uses sensors like silver-silver chloride electrodes, analog to digital converter components (sense electronics) like INTAN, TI and Nordic Nrf51822 component for data transfer to a host device. Sensors are connected to the analog to digital converters. Sense electronics components are connected to Nordic chip using standard interfaces like SPI / I2C. INTAN or TI electronics chipsets support multiple configurations that facilitate digitization of various physiological signals. Nordic Nrf51822, a highly flexible multi-protocol SOC, is used for near field communication allows of usage of multiple communication protocols on the same SOC. Implementation of such highly flexible architecture empowers to build systems by varying the combination of components themselves or by a mere change in configurations of the existing components. Figure 2 shows an EEG recording using TI ADS1299 as sense electronics chipset (analog to digital convertor) and Nordic Nrf51822 Chip Set for wireless communication. Mpu9150 Motion processor is to collect motion related data. The system uses Bluetooth Smart ${ }^{\circledR}$ (aka Bluetooth $4.0 \mathrm{LE})$ technology to transmit EEG data wirelessly in order to produce a portable, no-mess solution which is packaged entirely in an easy-to-wear visor that is more convenient to the user than most EEG systems available today.

Software design also plays a crucial role in efficiently utilizing modular hardware design. One way of developing such software is to mimic the hardware architecture. Modular hardware design mandates for a modular software design. Current software design uses a component-based software architecture to interact with the hardware efficiently. That puts forth the following requirements for the software design.

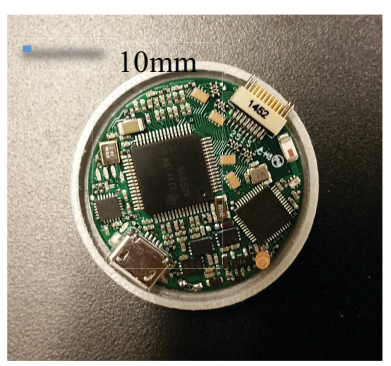

Figure 2. A prototype of small, low power wireless sensor platform (eight differential sensor channels and a nine-axis motion processor)

\section{Visual attention signal monitoring system}

EEG is a method of recording electromagnetic signals emitted by the brain that has enabled scientists and researchers to discover how to decode the mental states and intentions of a person in a noninvasive way. It has allowed for the understanding and characterization of a subject's brain signals, which are divided up into five primary frequency bands. These five primary frequency are Alpha (8-15 Hz), Beta (16-31 Hz), Gamma $(32+\mathrm{Hz})$, Theta $(4-7 \mathrm{~Hz})$, and Delta $(<4 \mathrm{~Hz})$. Well-established research in neuroscience has determined that activity in the $\alpha$ band is associated primarily with states of conscious quiet and rest, while activity in the $\beta$ band is associated primarily with conscious/alert mental activity such as thinking and receiving sensory stimulation [4].

Detecting brain waves from the head can be highly problematic due to the low amplitude of the signals (1-50 $\mu \mathrm{V}$ range) and the high impedance of the skin. The developed active sensor addresses these problems by acting as an impedance buffer, mitigating the noise and distortion at the skin/sensor interface. By placing the high impedance of the op-amp ( $\mathrm{G} \Omega$ range) in series with the relatively lower resistance of the skin $(100 \mathrm{k} \Omega)$, the resulting voltage-divider forces all the voltage to drop across the op-amp. In effect, next to zero noise power is generated at the skin and the SNR is greatly improved. Other features of the active sensor include a boost to the signal with $15 \mathrm{~V} / \mathrm{V}$ of gain, giving the signal added resistance to noise as it travels through wires and connectors into the ADC of the EEG board. The sensor also operates on very low power, with an operating current of around $1 \mu \mathrm{A}$ using a small coin battery for power.

The primary requirement is that the sensor must receive alpha and beta waves from the brain and deliver a differential output signal to the ADC on the EEG Board. The ADS1299 ADC has a resolution of about $.5 \mu \mathrm{V}$ while alpha and beta waves have amplitudes of $1-50 \mu \mathrm{V}$. With testing it was determined that $15 \mathrm{~V} / \mathrm{V}$ of gain provided by the active sensor is sufficient to complement the $24 \mathrm{~V} / \mathrm{V}$ programmable gain of the ADS1299, boosting the signal to an easily measurable level while not exceeding the limits of the supply rail. The frequency response was analyzed using a $20 \mathrm{mV}$ test signal from a 
signal generator. The voltage gain was measured at frequencies from 1-200 Hz. With a $-3 \mathrm{~dB}$ frequency of approximately $175 \mathrm{~Hz}$, the frequency range of interest (1$50 \mathrm{~Hz}$ ) remains free from significant attenuation. Also, this data shows that our $500 \mathrm{~Hz}$ sampling rate is sufficient to account for the major signal components, avoiding aliasing in software filtering. In the visual attention monitoring system, two sensors are placed at industry standard test points FP1 and FPZ [8]. An illustration of these points is given in Figure 3. FP1, in the frontal cortex, is commonly associated with concentration (beta waves), while FPZ is a signal reference point [4]. The earlobe was chosen as the standard reference for the sensors and EEG Board due to the weak signal at that location. Figure 4(b) shows our easy to wear headband style EEG system comparing with a GTECH 16-ch wireless EEG headset system (Fig. 4(a)).

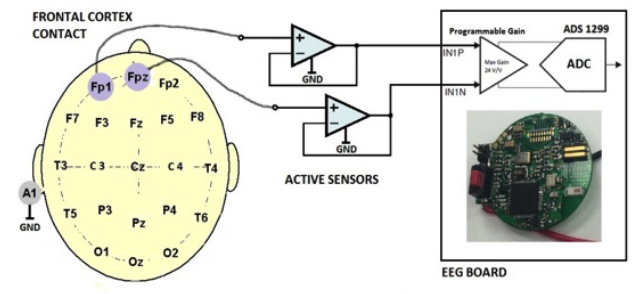

Figure 3. Sensor head placement of the EEG system.

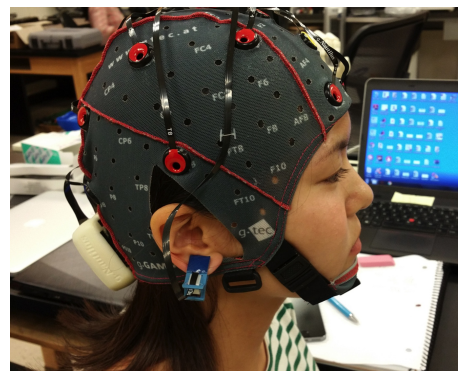

(a)

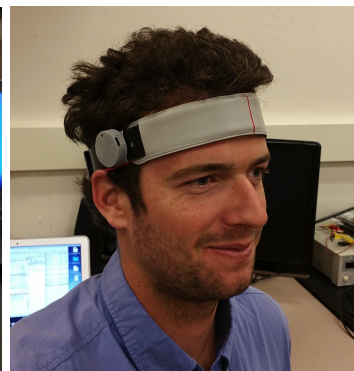

(b)
Figure 4. (a) Conventional EEG headset; (b) SDSU EEG headband.

The raw analog EEG signal obtained by the active sensor is digitized by the ADC of the EEG module. Once digitized, the data is transmitted wirelessly using a Nordic nRF1822 transceiver that follows the PAN technology defined within Bluetooth 4.0 LE standard. This technology is used to support our device's low power requirements while maintaining reliable communication between master/slave devices. The profile used to establish this communication link is known as GATT (Generic Attribute Profile); a master/slave link built from two machines sharing the same service characteristics.

\section{Experimental setup}

In our research, we use the machine learning to predict the concentration level of individual users, based on the results described in [4]. Our concentration detection algorithm consists of three main components. These include feature extraction, supervised machine learning, and generation of a concentration level using a mathematical model based on a predicted label. The supervised learning algorithm we are using is the support vector machine (SVM) technique [11] to provided high classification accuracy for a relatively small amount of features.

The frequency bands most closely related to the information of interest in are the alpha band, which is associated with relaxation, and the beta band, which is associated with concentration and deep thinking. In this, the features we extract from the signal are the energy in the alpha in beta bands, using the following equations:

$$
\begin{aligned}
& E_{\alpha}=\int_{8}^{13}|X(f)|^{2} d f \\
& E_{\beta}=\int_{14}^{30}|X(f)|^{2} d f
\end{aligned}
$$

The energy of the signal is taken every 250 samples, giving us one second worth of data at a $250 \mathrm{~Hz}$ sample rate. To implement this in software, we first found a signal processing library for $\mathrm{C \#}$, which would allow us to take the FFT of a signal. A wrapper class of our design encapsulates this library and contains all of the signal processing features that we need, including taking the FFT, IFFT, and finding the energy and power of a signal between two frequency bands. The decision to employ machine learning was based on the solutions in [4] and [6].

The method used in this system is support vector classification. Using this method, we first develop a training set for each user that is comprised of sample data from when the user is concentrating and when the user is relaxing. The energies of the alpha and beta bands are the features used in the training samples and as input to the support vector machine (SVM) is trained in order to predict if a user is concentrating or relaxing. SVMs work by solving an optimization problem in which a hyperplane is found that separates a set of labeled training data with the widest possible margin. The labels of the training data are known a priori, and nonlinear separations between the data are found using a kernel function that transforms the input data into a higher dimension. Figure 5 shows the experimental data collection procedures.

\subsection{Pre-training phase}

To train a support vector machine (SVM), we first have to obtain a corpus of data from the user with samples of them relaxing and concentrating. In this, we had to devise a way to induce concentration and relaxation. Based on prior research, it was shown that the users must perform some mental task to have a concentration induced [4]. Previous studies have used concentration tasks such as listening to English phrases and answering related questions [4]. 


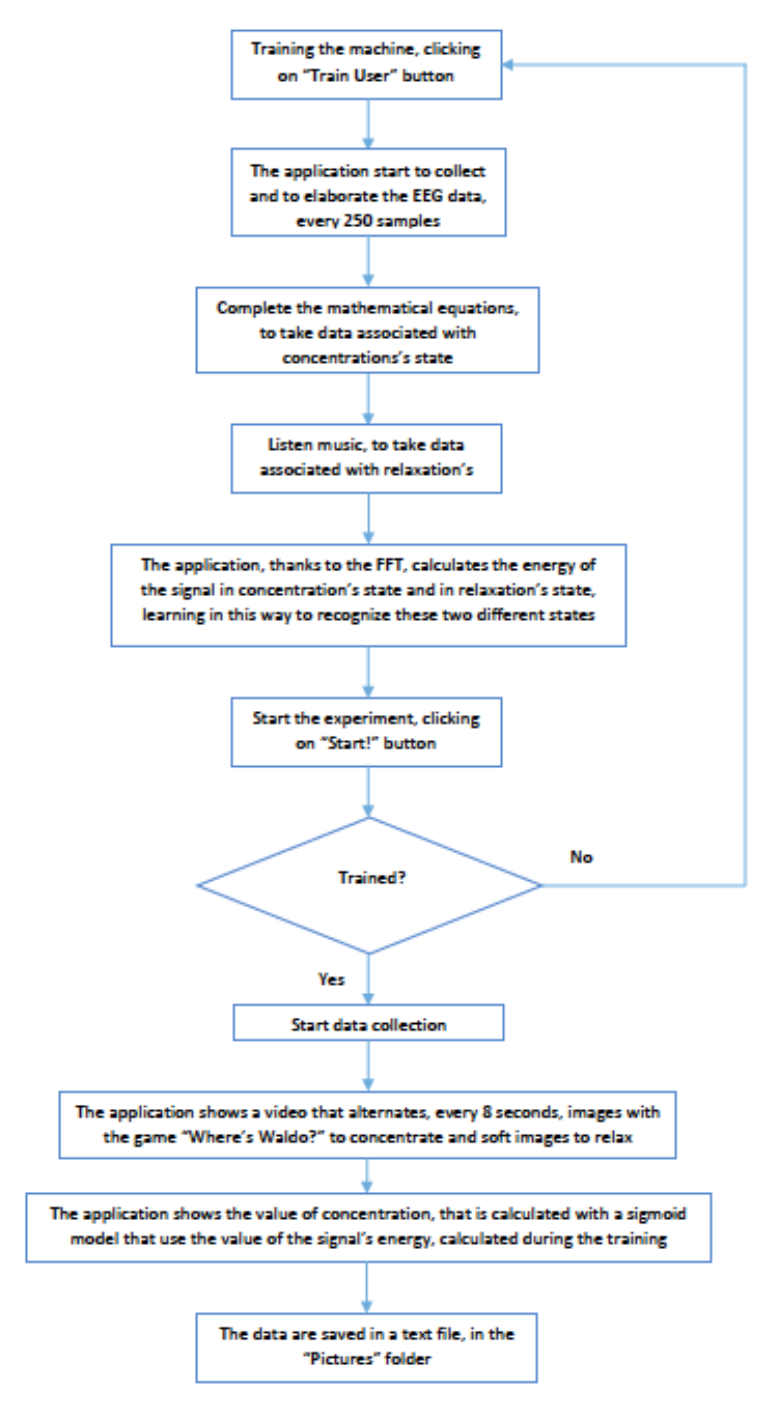

Figure 5. Experimental data collection procedures.

Since our application required a user perception of visual attention from images, we decided to have each user a training in the concentration phase by completing basic arithmetic in a timed setting. Next, the user would train the SVM for relaxation by selecting a 70 second relaxing sound clip from among 10 different options. During each of these training phases, the signal processing and machine learning components are collecting EEG training data by taking the energy of the signal in the alpha and beta bands and assigning a label of either "concentrating" or "relaxing" depending on the training phase. After the training phases and during the visual attention data collection, we use the predictions made by the support vector machine in conjunction with a mathematical model to determine a number between 0 and 1 that represents the user's instantaneous level of concentration. The model is based on the observation that energy in the beta frequency band $(14-30 \mathrm{~Hz})$ is generally higher when the user is concentrating versus when the user is relaxing. In this, we calculate an average of the user's beta energy during both the concentration and relaxation training phases to use as a benchmark.

\subsection{Visual attention signal collection}

During the actual experiment, we take the ratio of energy in the beta band at the current sample time with the average energy obtained during training, and supply this as the argument to a modified sigmoid function. The sigmoid function is given in Figure 6.

$$
\frac{1}{1+e^{-t}}
$$

The behavior of a sigmoid is such that it asymptotically approaches 0 as $\mathrm{t}$ approaches $-\infty$, and approaches 1 and $\mathrm{t}$ approaches $\infty$. Our modified implementation of the sigmoid function is given below:

$$
\begin{aligned}
& C_{i}=\frac{1}{1+e^{-\left(\frac{\beta_{i}}{\beta p_{i}}\right)}}-0.5 * p_{i} \\
& p_{i}=\left\{\begin{array}{lr}
0 & \text { Concentrating } \\
1 & \text { Relaxing }
\end{array}\right.
\end{aligned}
$$

Where $C_{i}$ is the concentration level, $p_{i}$ is the predicted label, $\beta_{i}$ is the energy in the beta band, and $\beta_{p i}$ is the average beta energy during training for either relaxation or concentration, depending on the prediction made by the SVM.

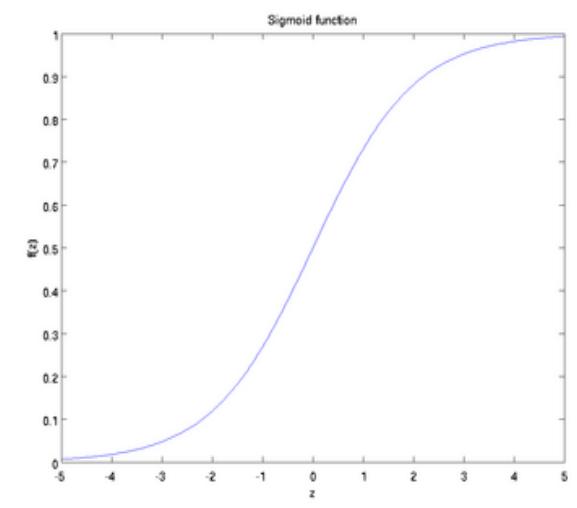

Figure 6. Sigmoid function for instantaneous concentrating level.

The experiment of detecting visual attention signal consists of a pair of test images to stimulate the test subject's EEG brain signal. The test images used for the analysis are shown in Figures 7-9. Each test consists of watching a video that contains the switching of the three sets of images of concentration and relaxation in series. As for the concentration visual stimulation purpose, we have chosen images from the game "Where's Waldo". The video starts with a countdown of six seconds, then eight seconds of concentration, eight seconds of relaxation, eight seconds of concentration, eight seconds of relaxation, eight seconds of concentration, and eight seconds of relaxation, respectively. First of all, the experimental procedure was verified with a GTECH EEG 
system (Figure 4(a)). Figure 10 shows the fast Fourier transform (FFT) of the signals from the GTECH EEG system. It can be noted that the fast Fourier transform (FFT) of the beta frequency band $(14-30 \mathrm{~Hz})$ from the "Where's Waldo" images has a higher FFT power than that from "Sunset" images.
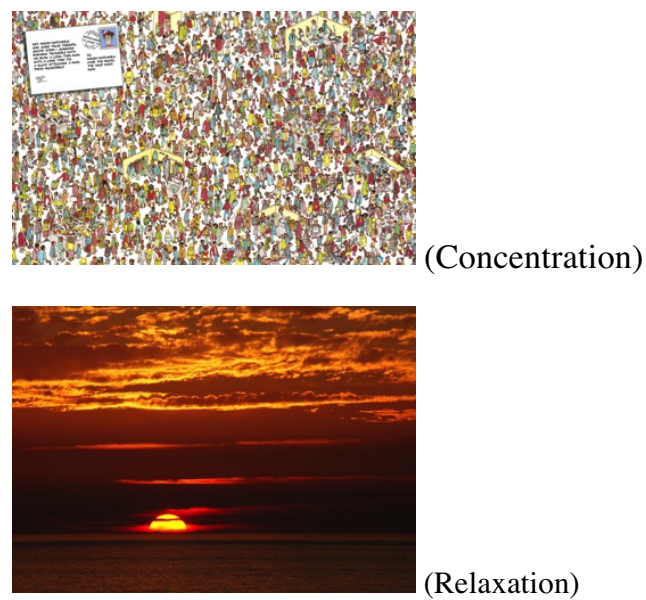

(Relaxation)

Figure 7. Visual attention test image pair \#1.
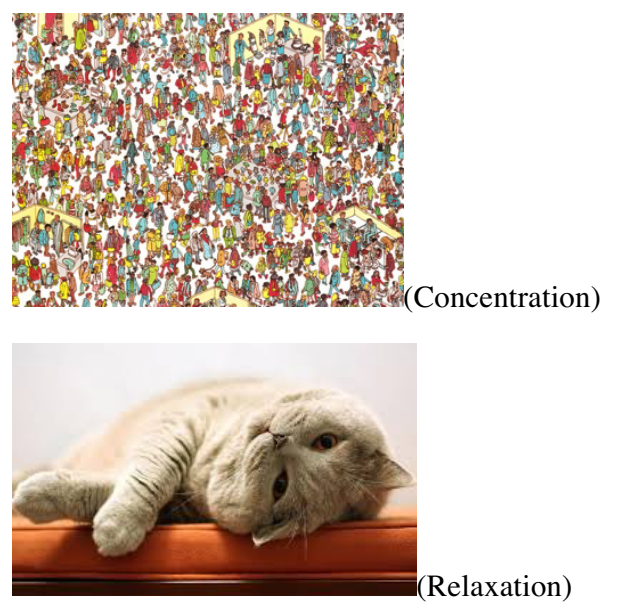

Figure 8. Visual attention test image pair $\# 2$.
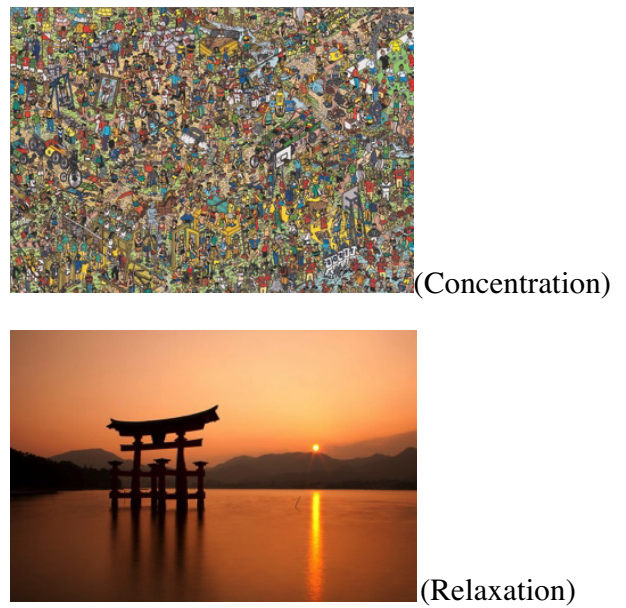

Figure 9. Visual attention test image pair \#3.

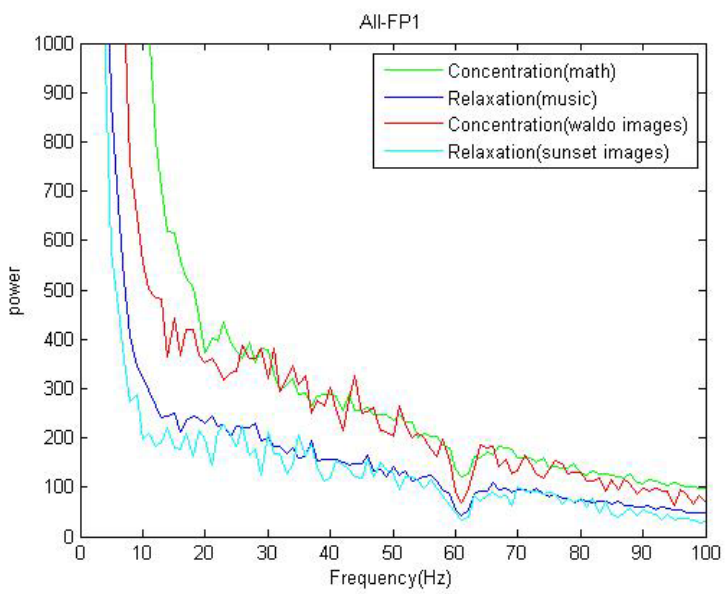

Figure 10. The fast Fourier transform (FFT) of the EEG signals (GTECH EEG system) from the test images.

We measured the instantaneous visual attention (concentration) level from three test subjects (not trained). The instantaneous visual attention was calculated using Equations (1)-(4) in process. For this study, total five sets of data from three test subjects have been recorded. For example, Figure 11 shows the recorded data set from the first test subject. From the figure, it is clear that there is a high-low pattern of signal intensity due to change of the images (i.e., concentration/relaxation).

Table 1 shows the average visual attention signal intensity for each image set. The results indicate that about $75 \%$ of tests recorded a higher average intensity from "Where's Waldo" images (i.e., concentration images). It was also found that a higher visual attention level was recorded by the test subjects after a training (i.e., several repetitions of the test procedure). Also, the accuracy of the data is dependent on several factors including wireless data transmission, the condition of electrodes, and a human factor (e.g., a lack of concentration due unwanted image, a motion artifact, etc.)

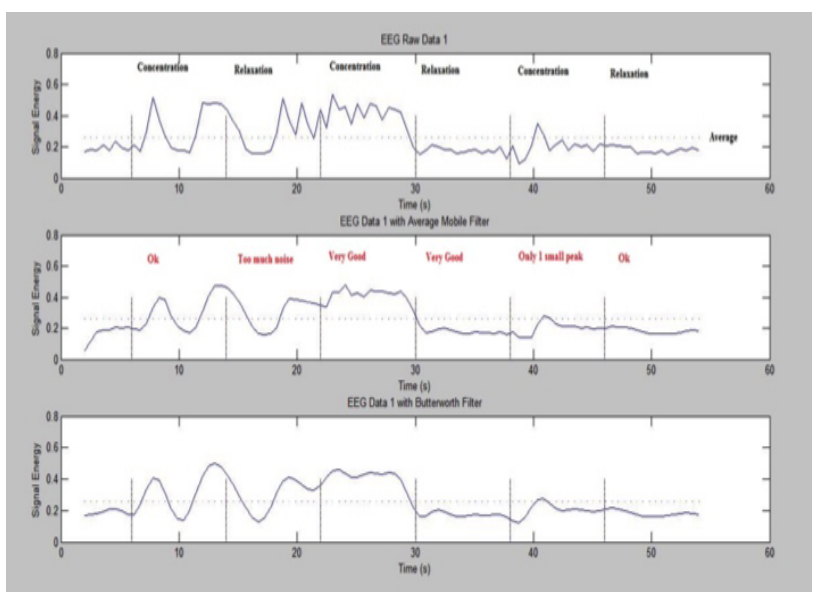

Figure 11. Recording visual attention signal (test data set from sample 1). 
Table 1. Average visual attention signal intensity data.

\begin{tabular}{|c|c|c|c|}
\hline $\begin{array}{l}\text { Image } \\
\text { set no. }\end{array}$ & $\begin{array}{l}\text { Sample } \\
\text { no. }\end{array}$ & $\begin{array}{l}\text { Average visual } \\
\text { attention signal } \\
\text { intensity - } \\
\text { concentration } \\
(0-1)\end{array}$ & $\begin{array}{l}\text { Average } \\
\text { visual } \\
\text { attention } \\
\text { signal } \\
\text { intensity - } \\
\text { relaxation } \\
(0-1)\end{array}$ \\
\hline 1 & 1 & 0.2053 & 0.1798 \\
\hline 1 & 2 & 0.2007 & 0.1962 \\
\hline 1 & 3 & 0.2575 & 0.2661 \\
\hline 1 & 4 & 0.2476 & 0.2071 \\
\hline 1 & 5 & 0.2368 & 0.2157 \\
\hline Average & & 0.2296 & 0.2130 \\
\hline 2 & 1 & 0.3894 & 0.1764 \\
\hline 2 & 2 & 0.3420 & 0.3070 \\
\hline 2 & 3 & 0.2443 & 0.2404 \\
\hline 2 & 4 & 0.2266 & 0.2355 \\
\hline 2 & 5 & 0.1831 & 0.1831 \\
\hline Average & & 0.2771 & 0.2285 \\
\hline 3 & 1 & 0.2053 & 0.1798 \\
\hline 3 & 2 & 0.2007 & 0.1962 \\
\hline 3 & 3 & 0.2575 & 0.2661 \\
\hline 3 & 4 & 0.2476 & 0.2071 \\
\hline 3 & 5 & 0.2368 & 0.2157 \\
\hline Average & & 0.2296 & 0.2130 \\
\hline
\end{tabular}

\section{Conclusions}

This paper presents a study in developing a visual attention (or concentration) detection system with an active sensor, Bluetooth communication with a host application, signal processing. The ability of the developed wireless sensor to accurately measure small signals by the brain has verified using standard test equipment. The digitization of this data and transmission over Bluetooth has been proven to be accurate by passing known signals over one of our EEG modules. Concentration prediction accuracy was validated through test subjects where we obtained accuracy in the range of $70-80 \%$. The culmination of this work is an engaging and novel system, which advances the future of mindcontrolled devices.

\section{Acknowledgment}

The research was partially funded by grants from the Korea Institute for Advancement of Technology (KIAT) and the Center for Sensorimotor Neural Engineering (CSNE) that is an NSF Engineering Research Center. We also thank SDSU CEREBRO team and Kei Nagano for their excellent work.

\section{References}

1. $<$ http://en.wikipedia.org/wiki/Electroencephalograph $\mathrm{y}>$

2. J. Jonides, Bulletin of the Psychonomic Society 21, 4: 247-50 (1983)

3. C. Eriksen, J. Hoffman, Perception \& Psychophysics 12, 2B: 201-4. (1972)

4. N.-H., Liu, C. Cheng-Yu, H.-C. Chu, Sensors, 13, 8: 10273-10286 (2013)

5. D.G. Duru, A.D. Duru, D.E. Barkana, O. Sanli, M. Ozkan, Neural Engineering (NER), 2013 6th International IEEE/EMBS Conference (2013)

6. L. George, F. Lotte, R.V. Abad, A. Lécuyer. Engineering in Medicine and Biology Society, EMBC, $2011 \quad$ Annual International Conference of the IEEE. IEEE (2011)

7. Q. Wang, S. Olga, M. K. Nguyen, Cyberworlds (CW), 2010 International Conference on. IEEE, (2010)

8. Y. Sakai, Y. Tohru, I. Wataru, Biomedical Engineering International Conference (BMEiCON), 2012. IEEE, (2012)

9. H. Brahim, Z. Haihong, G. Cuntai, W. Chuanchu, S.P. Kok, P.T. Keng, K.A. Kai, Circuits and Systems, 2009. ISCAS 2009. IEEE International Symposium on. IEEE, (2009)

10. T. Shanbao, N.V. Thakor, Quantitative EEG Analysis Methods and Applications (London: Artech House, 2009)

11. $<$ https://en.wikipedia.org/wiki/Support_vector_mach ine $>$ 